\title{
Aprendizagem baseada em projetos num curso de técnico superior profissional de desenvolvimento de software
}

\section{Project-based learning in a higher professional technical course in software development}

\author{
Paulo Alves* \\ Carlos Morais $^{* *}$ \\ Luísa Miranda ${ }^{* * *}$
}

\section{Resumo}

Considerando que o foco do processo de ensino aprendizagem deve estar mais centrado na aprendizagem do que no ensino, surge a necessidade de fomentar estratégias assentes em metodologias que tornem o estudante o ator principal da sua aprendizagem, construindo aprendizagens significativas que possam responder a problemas reais da sociedade onde se encontra integrado. Os principais objetivos deste artigo consistem em fundamentar estratégias ativas de aprendizagem no desenvolvimento de competências, suportadas por metodologias baseadas em projetos, e apresentar de forma fundamentada o modelo de um curso de técnico superior profissional de desenvolvimento de software. A metodologia utilizada é de natureza qualitativa, com procedimentos associados à pesquisa bibliográfica e uma abordagem reflexiva e interpretativa. Tendo presentes as necessidades da sociedade e os objetivos de formação dos cursos de técnico superior profissional na área das Ciências Informáticas defende-se a utilização de metodologias ativas de aprendizagem com forte ligação à prática, entre as quais a aprendizagem baseada em projetos. Como resultados da investigação apresenta-se uma reflexão sobre as metodologias de aprendizagem baseadas em projetos e o exemplo do plano de um curso de técnico superior profissional de desenvolvimento de software, em funcionamento numa instituição portuguesa de ensino superior público. Este curso de técnico superior profissional foi desenhado para responder à necessidade de técnicos superiores no domínio da Informática e com a finalidade de ser desenvolvido e avaliado em função da adoção de metodologias de aprendizagem baseadas em projetos no seu funcionamento.

Palavras-chave: Aprendizagem baseada em projetos. Cursos de técnico superior profissional. Curso de desenvolvimento de software. Competências profissionais. Aprendizagem ativa.

Recebido em 07/10/2018 - Aprovado em 03/02/2019

http://dx.doi.org/10.5335/rep.v26i2.8729

Doutor em Tecnologias e Sistemas de Informação pela Universidade do Minho, Portugal. Professor Adjunto do Departamento de Informática e Comunicações da Escola Superior de Tecnologia e Gestão do Instituto Politécnico de Bragança. É membro integrado do Centro de Investigação em Digitalização e Robótica Inteligente, Portugal. Email: palves@ipb.pt

** Agregado em Educação, especialidade de Educação a Distância e Elearning, Universidade Aberta, Portugal. Doutor em Educação, área de Conhecimento de Metodologia do Ensino da Matemática, Universidade do Minho. Professor adjunto na Escola Superior de Educação do Instituto Politécnico de Bragança. É membro integrado do Centro de Investigação em Estudos da Criança da Universidade do Minho, Portugal. Email: cmmm@ipb.pt

*** Doutora em Educação na área de conhecimento de Tecnologia Educativa pela Universidade do Minho, Portugal. Professora Adjunta da Escola Superior de Tecnologia e Gestão, do Instituto Politécnico de Bragança, Portugal. Email: Imiranda@ipb.pt 


\title{
Abstract
}

\begin{abstract}
Considering that the focus of the teaching and learning process must be centred on learning rather than teaching, the need arises to foster strategies based on methodologies which may turn students into the main actors of their learning, thus building meaningful learning experiences that may respond to real problems faced by the society they integrate. The main aims of this paper are to ground active learning strategies for skill development anchored in project-based methodologies and to present a substantiated model of a higher professional technical course in Software Development. This research follows a qualitative methodology, with procedures associated with bibliographic research and a reflexive and interpretive approach. Bearing in mind the society needs as well as the training goals of the higher professional technical courses in the field of IT sciences, we advocate the use of active learning strategies strongly connected to practice, among which the project-based learning. The results of this study consist of a reflection on project-based learning methodologies and of a study programme example of a higher professional technical course in Software Development running in a Portuguese public higher education institution. This higher professional technical course was designed to respond to a demand for higher technicians in the area of IT, and also with the aim to be developed and assessed with regard to the adoption of project-based learning methodologies throughout its operationalisation.
\end{abstract}

Keywords: Project-Based Learning. Higher Professional Technical Courses. Course in Software Development. Professional Skills. Active Learning.

\section{Introdução}

Cada vez é mais difícil prever o futuro a curto e a longo prazo. Esta incerteza implica reflexões profundas nas formações oferecidas pelas instituições de ensino superior, bem como nas metodologias de aprendizagem que devem ser fomentadas com os estudantes, para que estes, depois de adquiridas as suas formações, possam responder com êxito aos desafios do século XXI.

Atendendo ao forte interesse que a área de programação de computadores tem a nível nacional e internacional e à importância que as metodologias de aprendizagem associadas à prática têm no ensino superior, a instituição a que os investigadores deste artigo pertencem propôs a criação do curso técnico superior profissional de desenvolvimento de software. Este curso encontra-se no início do 2..$^{\circ}$ ano de funcionamento e está integrado num projeto piloto a nível nacional, da iniciativa INCoDe.2030 do Governo de Portugal. Dos objetivos deste curso sobressai a necessidade de formar profissionais na área da programação de computadores, com competências para responderem às necessidades do mundo real e poderem fomentar projetos de intervenção na comunidade. No desenvolvimento do curso aposta-se em metodologias ativas orientadas para a prática, nomeadamente aprendizagem baseada em projetos.

Neste artigo é apresentada a fundamentação de metodologias de aprendizagem baseadas em projetos, bem como a contextualização dos cursos de técnico 
superior profissional e a caracterização do curso superior profissional de desenvolvimento de software que constitui um exemplo de um curso em funcionamento, desenhado com a finalidade de poderem ser testadas e avaliadas metodologias ativas de aprendizagem baseadas na prática.

O artigo está organizado nas seguintes secções principais: Introdução, Aprendizagem baseada em projetos no desenvolvimento de competências profissionais, Cursos de técnico superior profissional (CTeSP), Curso de técnico superior profissional em desenvolvimento de software com metodologias baseadas em projetos, Considerações finais e Bibliografia.

\section{Aprendizagem baseada em projetos no desenvolvimento de competências profissionais}

\section{Aprendizagem ativa e as competências para o século XXI}

$\mathrm{Na}$ sociedade do conhecimento as organizações operam numa economia global caracterizada pela competição, interdependência económica e colaboração. Esta economia, assim como as rápidas alterações a que está sujeita, estão muito dependentes das tecnologias de informação e comunicação (TIC), no entanto as TIC não criam uma economia baseada no conhecimento (LAAR et al., 2017).

$\mathrm{O}$ mercado de trabalho exige profissionais altamente qualificados, nomeadamente, no domínio das TIC. Para preencher essa exigência, uma abordagem importante consiste em estabelecer uma ponte entre os aspetos teóricos abordados nos cursos formais e as competências técnicas necessárias no cotidiano do mundo profissional (MARTINS et al., 2017).

A aprendizagem ativa desloca o foco do professor e a distribuição dos conteúdos do curso para o estudante, assim como o envolvimento ativo deste com o material, através de técnicas de aprendizagem ativa e modelagem por parte do professor, fazendo com que os estudantes deixem de ter o papel de recetores passivos e aprendam a praticar como apreender conhecimentos e habilidades, e a usá-los de forma significativa (FLORIDA STATE UNIVERSITY, 2011).

Quando se pensa em metodologias ativas orientadas para o desenvolvimento de competências associadas à prática e com elevado nível de exigência é importante ter presente que as competências sofrem diversos níveis de importância dependentes da época e do contexto. Assim, num relatório da World Economic Forum (2016) 
evidenciam-se as 10 competências consideradas mais importantes para o mercado de trabalho em 2015, ao mesmo tempo que se infere sobre as competências mais importantes em 2020, conforme se evidencia na Tabela 1.

Tabela 1 - Top 10 das competências para o mercado de trabalho em 2015 e 2020

\begin{tabular}{|c|c|c|}
\hline Ordenação & Competências em 2020 & Competências em 2015 \\
\hline 1..$^{a}$ & Resolução de problemas complexos & Resolução de problemas Complexos \\
\hline 2. ${ }^{\mathrm{a}}$ & Pensamento crítico & Colaboração \\
\hline 3. ${ }^{\mathrm{a}}$ & Criatividade & Gestão de recursos humanos \\
\hline 4..$^{\mathrm{a}}$ & Gestão de recursos humanos & Pensamento crítico \\
\hline 5.. & Colaboração & Negociação \\
\hline 6..$^{a}$ & Inteligência emocional & Controlo de qualidade \\
\hline 7.. & Tomada de decisões & Interajuda \\
\hline 8..$^{\mathrm{a}}$ & Interajuda & Tomada de decisões \\
\hline 9. & Negociação & Ouvir os outros \\
\hline $10 .{ }^{\mathrm{a}}$ & Flexibilidade cognitiva & Criatividade \\
\hline
\end{tabular}

Fonte: adaptado de World Economic Forum (2016).

Pela observação da Tabela 1 , constata-se que a competência associada à resolução de problemas complexos mantem-se inalterada de 2015 para 2020, no entanto, atendendo à ordenação apresentada o pensamento crítico e a criatividade estão a assumir uma tendência crescente de importância.

Considerando que na análise de cada uma das competências terá de se ter sempre presente a tecnologia em que o mundo se encontra envolvido e a permanente necessidade de mudança a que diariamente se assiste e com base no relatório de 2016 do World Economic Forum - Future of Jobs Report, segue-se uma breve referência a cada uma das 10 competências referidas para 2020:

- resolução de problemas complexos: a tecnologia, geralmente, facilita a resolução de problemas, no entanto, também pode aumentar a complexidade das situações com que o ser humano tem de lidar, exigindo-se a cada pessoa que seja capaz de viver com diversas e múltiplas dimensões, e resolver problemas complexos;

- pensamento crítico: cada vez mais é necessário questionar as ações realizadas, ou que se pretendam realizar. Muitos dos gestos diários do ser humano 
são realizados de forma automática ou mediados pela tecnologia. Neste sentido, o ser humano deve ser dotado de competências que lhe permitam utilizar o raciocínio e o pensamento lógico. É imperioso aproveitar os benefícios que as máquinas e a tecnologia proporcionam, mas tendo sempre presente princípios que tenham em conta a ética e o bom relacionamento social;

- criatividade: diariamente surgem novos serviços e novos produtos. Os empregadores veem-se confrontados com a necessidade de manterem nas suas empresas pessoas familiarizadas com as tecnologias, para poderem utilizar os seus conhecimentos em novos produtos, novos serviços e novas formas de se relacionarem e cativar o seu público alvo;

- gestão de recursos humanos: é importante desenvolver competências que permitam motivar pessoas, desenvolver os seus talentos e habilidades, e selecionar as mais adequadas e que se sintam mais confortáveis em cada função;

- colaboração: é importante promover competências sociais, no sentido de cada pessoa desenvolver o poder de colaborar, ajustar-se em relação aos outros e ser sensível às necessidades dos outros;

- inteligência emocional: os empregadores podem focar o seu interesse na contratação de pessoas que estão atentas às reações dos outros e ao impacto das suas reações;

- tomada de decisões: as organizações lidam cada vez com maior quantidade de dados. Esta quantidade de dados implica a necessidade de trabalhadores com competências para analisá-los corretamente e usá-los na tomada de decisões inteligentes;

- interajuda: competência que se traduz na permanente procura de formas de ajudar os outros;

- negociação: competência com aplicação a diversos ramos de atividade, tornando-se uma competência muito valorizada, nomeadamente para trabalhos que envolvam computação, matemática, análise de dados e desenvolvimento de software;

- flexibilidade cognitiva: envolve criatividade, raciocínio lógico e sensibilidade para compreender e resolver problemas.

Quando os estudantes aprendem ativamente retêm mais conteúdo, por mais tempo e podem aplicar o que aprendem a maior variedade de contextos. Muitos 
docentes assumem que o seu papel é ensinar, enquanto deveriam pensar que o seu papel é ajudar os estudantes a aprender. Todas as técnicas de aprendizagem ativa destinam-se a ajudar os estudantes a fazer conexões relevantes entre os materiais do curso, transformar os materiais do curso assentes em linguagem opaca ou ideias em algo que os estudantes possam integrar no seu próprio conhecimento e na memória de longo prazo (FLORIDA STATE UNIVERSITY, 2011).

\section{Aprendizagem baseada em projetos}

Aprendizagem ativa é uma metodologia em que os estudantes deixam de ser simples espetadores, uma vez que participam, experimentam e encaram a sua própria trajetória.

Existem várias formas de implementar a aprendizagem ativa, entre elas a Aprendizagem Baseada em Projetos (PBL), a qual tem vindo a ser aplicada com sucesso em diversas áreas (SEMANA; HAUSMANNB; BEZERRA, 2018).

A aprendizagem baseada em projetos tem vindo a ser adotada em diversos contextos educacionais (MARTINS et al., 2017), incluindo no currículo de engenharias, quer para aumentar a motivação, quer para permitir que os estudantes apliquem na prática o que aprenderam (NIEH; CHOU, 2018; REQUIES et al., 2018). Este modelo tem vindo a assumir-se como um dos principais modelos pedagógicos para responder aos desafios do novo século (BENDER, 2012).

Regularmente, a metodologia híbrida, Aprendizagem Baseada em Problemas e em Projetos, tem sido utilizada na área de Ciências da Computação aplicada a problemas / projetos, trabalhada por grupos de estudantes e facilitada por tutores (MARTINS et al., 2017).

Segundo Krajcik e Blumenfeld (2006), a aprendizagem baseada em projetos permite que os estudantes aprendam fazendo e aplicando ideias. Os estudantes envolvem-se em atividades do mundo real que são semelhantes às atividades com que os profissionais são confrontados. Bender (2012) refere que o uso de projetos autênticos, com base em uma questão, tarefa ou problema é altamente motivador e envolvente, para ensinar aos estudantes os conteúdos académicos aplicados ao contexto de trabalho.

A aprendizagem baseada em projetos permite aos estudantes investigar questões, propor hipóteses e explicações, discutir as suas ideias, estimular o aparecimento de novas ideias e experimentar essas ideias. Exige que os estudantes projetem e concluam projetos, em contextos que são confrontados com diversos 
problemas abrangentes para os quais precisam de encontrar soluções. A resolução de problemas é um processo demorado e, quando necessário, para completar uma tarefa em aprendizagem baseada em projetos, exige mais tempo do estudante do que métodos passivos de aprendizagem, porque os estudantes, geralmente, precisam de fazer várias tentativas antes de atingirem uma conclusão satisfatória (MAHASNEH; ALWAN, 2018).

Os estudantes que aprendem por este método tornam-se criativos e construtivos, dada a gama quase ilimitada de projetos, desde criar um portfólio de aprendizagem, construir um modelo a partir de um esquema, produzir um vídeo ou criar um website, as possibilidades são infinitas (MAHASNEH; ALWAN, 2018).

Os estudantes devem ser preparados para propor, investigar e desenvolver soluções para os problemas que possam surgir na sua vida profissional. Devem ser estimulados durante o seu curso a trabalhar com problemas com níveis crescentes de complexidade, o que exige dos docentes uma mudança nas metodologias utilizadas.

A metodologia de aprendizagem baseada em projetos permite desenvolver competências dos estudantes a diversos níveis (RIBEIRO; MIZUKAMI, 2004):

- conhecimentos (hard skills): ciência e tecnologia, programação, administração, impactos ambientais e sociais da tecnologia, entre outros;

- habilidades (soft skills): desenvolvimento de projetos, resolução de problemas, comunicação, trabalho em equipa, autoavaliação e avaliação por pares, entre outros;

- atitudes (soft skills): ética, responsabilidade para com os colegas, sociedade e profissão, iniciativa, flexibilidade, empreendedorismo, motivação para a aprendizagem ao longo da vida, entre outras.

Dragoumanos, Kakarountas e Fourou (2017) reforçam a importância que a aprendizagem baseada em projetos tem para os estudantes na aquisição de competências necessárias no século XXI, nomeadamente, adquirirem conhecimento e desenvolverem competências trabalhando em equipa por um período prolongado de tempo, num tema específico. Para desenvolverem o projeto necessitam de aprender a fazer gestão do tempo, ter espírito de equipe e competências associadas à comunicação.

O modelo de aprendizagem baseada em projetos desenvolvido pelo Buck Institute for Education (LARMER; MERGENDOLLER; BOSS, 2015) centra-se no estudante, estando na base os objetivos de aprendizagem e os conhecimentos chave. 
Para atingirem esses objetivos é necessária a aquisição de competências orientadas ao sucesso. Os estudantes necessitam de ser capazes de mobilizar os seus conhecimentos para pensar e analisar as questões atuais, resolver novos problemas e contribuir para o diálogo cívico.

Os elementos essenciais para o desenho dos projetos, tendo em conta os objetivos de aprendizagem dos estudantes, são: problema ou questão, questionamento sustentado, autenticidade, voz e escolha do estudante, reflexão, crítica e revisão, e ser um produto público. Segue-se breve descrição dos elementos referidos:

- problema ou questão: problemas e perguntas fornecem a estrutura de organização para o projeto e tornam a aprendizagem significativa, porque dão um propósito à aprendizagem. Ao focar o processo num problema ou pergunta, os estudantes não só dominam novos conhecimentos, mas também aprendem quando e como esse novo conhecimento pode ser usado;

- questionamento sustentado: a investigação exerce forte influência no desenvolvimento do projeto. Problemas ou perguntas são usadas para iniciar a investigação. Questões como: “O que sabemos?" e "O que precisamos saber?" levam os estudantes a identificar o processo de resolução do problema e a responder à pergunta que orienta a investigação. A resposta a uma questão inicial pode levantar novas questões, iniciando-se novo processo, levando a um aprofundamento da investigação;

- autenticidade: a autenticidade permite não só atribuir um significado ao projeto, como também aumentar a motivação para a sua realização;

- voz e escolha do estudante: diante de um problema ou questão desafiadora os estudantes devem ser capazes de avaliar e tomar decisões sobre como resolvê-los. Os estudantes ao expressarem as suas ideias e ao fazerem escolhas ao longo do projeto terão consequências positivas, tanto para a aprendizagem como para a sua motivação;

- reflexão: os estudantes e o professor precisam de refletir ao longo de todo o projeto sobre a eficácia das suas atividades de investigação, a qualidade do trabalho, os obstáculos enfrentados e como eles podem ser superados. Estas reflexões mantêm o projeto no caminho certo;

- crítica e revisão: o papel dos estudantes não é o de, simplesmente, realizarem tarefas decididas pelos professores, mas gerir ativamente e entender os resultados da aprendizagem. Isso inclui avaliarem o seu próprio progresso, 
serem mais responsáveis pela sua aprendizagem, e estarem envolvidos com os colegas a aprender de forma colaborativa;

- produto público: a aprendizagem baseada em projetos oferece a oportunidade aos estudantes de criarem um produto e compartilhá-lo com o público para além da sala de aula. Isso tem várias consequências positivas, nomeadamente incentiva os estudantes a fazerem melhor, aumentando a motivação e o seu envolvimento.

A aprendizagem baseada em projetos refere-se ao envolvimento dos estudantes na realização de um projeto no contexto do mundo real, através do qual se movem em direção ao desenvolvimento de conhecimentos e habilidades relacionadas com o projeto (CAVANAUGH, 2004).

$\mathrm{O}$ foco nas experiências de aprendizagem autênticas que os estudantes podem realizar no mundo real é uma característica de praticamente todas as experiências de aprendizagem baseada em projetos, que aumenta a motivação dos estudantes para participarem ativamente nos projetos (BENDER, 2012).

Os estudantes envolvidos na aprendizagem baseada em projetos obtêm notas mais altas do que os estudantes que frequentam aulas tradicionais (MARX et al., 2004; RIVET; KRAJCIK, 2004).

A motivação com este tipo de metodologia ativa é maior que com a tradicional, o trabalho em equipa melhora o relacionamento entre os estudantes, o diálogo entre os estudantes e o professor é mais fluido, e todos esses fatores levam a um ambiente de trabalho construtivo no qual os estudantes supervisionam o seu próprio processo de aprendizagem (REQUIES et al., 2018).

A aprendizagem baseada em projetos afasta-se do modelo tradicional centrado no professor. Em vez disso, os estudantes são incentivados a trabalhar e a aprender de forma independente (CHOUNTA; MANSKE; HOPPE, 2017), adquirindo novas competências para um mercado de trabalho mais exigente e em rápida evolução.

\section{Cursos de técnico superior profissional (CTeSP)}

O reconhecimento da necessidade de tornar cada mais próximas a teoria e a prática, bem como de dar resposta às necessidades reais das pessoas faz com que os países adequem as suas formações aos contextos temporais, tecnológicos e sociais. Neste sentido, em vários países, entre os quais Portugal, estão a ser fomentados cursos de técnicos superiores profissionais em instituições de ensino superior. 
O regime jurídico português do curso de técnico superior profissional está previsto no Decreto-Lei n.․ 74/2006, de 24 de março, alterado e republicado pelo Decreto-Lei n. ${ }^{\circ}$ 63/2016, de 13 de setembro, que no seu artigo $40^{\circ}$ - A afirma: o diploma de técnico superior profissional é conferido aos que demonstrem:

a) possuir conhecimentos e capacidade de compreensão numa área de formação, e a um nível que: i) Sustentando-se nos conhecimentos de nível secundário, os desenvolva e aprofunde; ii) Se apoie em materiais de ensino de nível avançado e lhes corresponda; iii) Constitua a base para uma área de atividade profissional ou vocacional, para o desenvolvimento pessoal e para o prosseguimento de estudos com vista à conclusão de um ciclo de estudos conducente à atribuição do grau de licenciado;

b) saber aplicar, em contextos profissionais, os conhecimentos e a capacidade de compreensão adquiridos;

c) ter capacidade de identificar e utilizar informação para dar resposta a problemas concretos e abstratos bem definidos;

d) possuir competências que lhes permitam comunicar acerca da sua compreensão das questões, competências e atividades, com os seus pares, supervisores e clientes;

e) possuir competências de aprendizagem que lhes permitam prosseguir estudos com alguma autonomia.

Por outro lado, a Direção Geral do Ensino Superior (português) esclarece que o curso de técnico superior profissional não confere grau académico e a conclusão, com aproveitamento, atribui o diploma de técnico superior profissional. Este ciclo de estudos é ministrado no ensino politécnico, tem 120 ECTS (European Credit Transfer and Accumulation System) e a sua duração é de quatro semestres curriculares de trabalho dos estudantes, constituídos por um conjunto de unidades curriculares organizadas em componentes de formação geral e científica, formação técnica e formação em contexto de trabalho, que se concretiza através de um estágio.

Os titulares do diploma de técnico superior profissional podem aceder e ingressar nos ciclos de estudos de licenciatura e de mestrado integrado através de um concurso especial próprio a si destinado, adquirindo o respetivo grau académico.

Pelas características referidas os CTeSP são cursos adequados para responderem num curto período de tempo a necessidades de formação nas mais variadas áreas de que a sociedade careça, em particular nos domínios das tecnologias de informação e comunicação, área que em poucos anos ocupou um enorme espaço na 
vida das pessoas tornando-se a sua presença permanente e o seu uso imprescindível em qualquer sociedade.

A importância atribuída à formação associada às tecnologias digitais é evidenciada pela Comissão Europeia, atendendo às várias iniciativas que promove com a finalidade de aumentar a formação em competências digitais, nomeadamente, para responder às necessidades do mercado de trabalho, necessidades dos consumidores, modernizar a educação e antecipar a necessidade de novas competências. A nova Agenda de Competências para a Europa (COMISSÃO EUROPEIA, 2016) tem por missão reforçar o capital humano, a empregabilidade e a competitividade, apresentando uma série de ações e iniciativas com a ambição de combater o défice de competências digitais na Europa.

A estabilidade no emprego já deixou de estar ligada a um local geográfico ou a uma determinada empresa, estando muito mais associada às competências que cada pessoa possui para se adaptar à mudança e às necessidades do mercado de trabalho em cada momento. Para Boyaci e Atalay (2016) o local de trabalho atual requer trabalhadores que possam encontrar, processar e estruturar informações, que possam resolver problemas, que sejam inovadores e criativos, e que possuam capacidades de comunicação e cooperação.

De acordo com Voogt e Roblin (2012), o conhecimento tornou-se vital no século XXI e as pessoas necessitam de adquirir competências para ingressarem no mercado de trabalho exigidas pelo século XXI, ou seja, necessitam de competências que incluam colaboração, comunicação, alfabetização digital, cidadania, resolução de problemas, pensamento crítico, criatividade e produtividade.

As tecnologias de informação e comunicação continuam a provocar mudanças tecnológicas e sociais com velocidades imprevisíveis exigindo permanentemente novas necessidades e novas competências. Neste sentido, Binkley et al. (2012) apresentam as seguintes categorias de competências para o século XXI: modos de pensar (criatividade e inovação, pensamento crítico, resolução de problemas e tomada de decisão, aprender a aprender e metacognição); formas de trabalho (comunicação, colaboração e trabalho em equipe); ferramentas para trabalhar (literacia da informação, tecnologias da informação e alfabetização em comunicação); e modos de viver (vida e carreira, responsabilidade pessoal e social).

Conscientes das dificuldades que as rápidas mudanças sociais e tecnológicas provocam em termos das necessidades de novos profissionais e de adequação dos existentes para o mundo do trabalho, com competências cada vez mais exigentes para o século XXI, nomeadamente na área de Ciências Informáticas, a instituição 
a que os investigadores pertencem propôs o curso de técnico superior profissional de Desenvolvimento de Software, o qual se encontra em funcionamento numa instituição portuguesa de ensino superior público, desde o ano letivo de 2017/2018.

\section{Curso de técnico superior profissional em desenvolvimento de software com metodologias baseadas em projetos}

\section{Contexto de desenvolvimento do curso de técnico superior profissional de desenvolvimento de software}

A programação de computadores é considerada uma área de grande interesse, quer a nível académico, quer a nível económico e social. Assiste-se a vários projetos em todos os níveis de ensino, assim como se assiste a resultados da aplicação da programação e de software em grande parte dos atos diários, nomeadamente, listas de espera, controlo de presenças, operações bancárias, contabilidade, viagens e a grande parte das atividades das empresas e das instituições. Neste sentido, a formação de técnicos superiores profissionais nesta área é da maior importância, pois de acordo com dados da Comissão Europeia, existe um elevado défice de profissionais da área das TIC, estimando-se que poderão surgir 500000 vagas por preencher para profissionais de TIC até 2020 (COMISSÃO EUROPEIA, 2017).

O curso técnico superior profissional em desenvolvimento de software que se encontra no segundo ano de funcionamento, e no qual se espera experimentar novas metodologias assentes na prática, depois de avaliado poderá constituir um modelo de curso para poder ser ampliado a nível nacional e internacional, pois este curso está integrado na Iniciativa Nacional em Competências Digitais e.2030, Portugal INCoDe.2030, ${ }^{1}$ que tem por objetivos reforçar competências digitais, garantindo a literacia digital e a inclusão digital para o exercício pleno da cidadania, estimular a especialização em tecnologias e aplicações digitais para a qualificação do emprego e uma economia de maior valor acrescentado, e produzir novos conhecimentos em cooperação internacional.

A Iniciativa Nacional em Competências Digitais e.2030 é orientada a partir dos seguintes eixos e respetivos objetivos: inclusão - assegurar a generalização do acesso equitativo às tecnologias digitais a toda a população para obtenção de informação, comunicação e interação; educação - assegurar a educação das camadas mais jovens da população através do estímulo e reforço nos domínios da literacia 
digital e das competências digitais em todos os ciclos de ensino e de aprendizagem ao longo da vida; qualificação - capacitar profissionalmente a população ativa dotando-a dos conhecimentos necessários à integração num mercado de trabalho que depende fortemente de competências digitais; especialização - promover a especialização em tecnologias digitais e aplicações para a qualificação do emprego e a criação de maior valor acrescentado na economia; investigação - garantir as condições para a produção de novos conhecimentos e a participação ativa em redes e programas internacionais de I\&D.

O desenho do curso de técnico superior profissional de desenvolvimento de software enquadra-se nos eixos Educação e Qualificação. O curso situa-se no âmbito da Educação porque com ele pretende-se a promoção da inovação pedagógica nos processos de ensino e aprendizagem, e o reforço de competências analíticas e críticas através da promoção de projetos e práticas pedagógicas no âmbito da lógica, algoritmos e programação, ética aplicada ao ambiente digital, literacia para os media na era digital e cidadania na era digital. Também se pode considerar enquadrado no eixo da Qualificação porque integra uma rede de oferta nacional de cursos de curta duração a nível profissional e superior que permite melhorar as competências digitais existentes e a criação de novas competências.

A metodologia privilegiada para o desenvolvimento do curso é a aprendizagem baseada em projetos, que de acordo com Iturregi et al. (2017), entre os diferentes campos de conhecimento, a aprendizagem baseada em projetos mostrou-se eficaz na educação e em engenharia.

A melhoria de competências e a criação de novas competências implicam grande esforço dos professores, e como sugere Kennedy (2007), desenvolver ligações entre os resultados de aprendizagem, atividades de ensino e aprendizagem e avaliação.

Na Figura 1, apresentam-se aspetos cuja adequada combinação enfatiza a complexidade do processo de ensino e aprendizagem, pois à multiplicidade de resultados de aprendizagem em qualquer um dos níveis cognitivo, afetivo e psicomotor é possível associar um vasto e diversificado conjunto de atividades de aprendizagem, bem como múltiplas formas de avaliação. 
Figura 1: Resultados de aprendizagem, atividades de ensino e aprendizagem e avaliação<smiles>C1=CCC=C1</smiles>

Resultados de aprendizagem

Cognitivos

Evidenciar:

- Conhecimento

- Compreensão

- Aplicação

- Análise

- Síntese

- Avaliação

Afetivos

- Integração de convicções, ideias e atitudes

Psicomotores

- Aquisição de competências

físicas

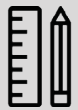

Atividades de ensino e aprendizagem

- Palestras

- Tutoriais

- Seminários

- Discussão de temas

- Colocação de questões desafiantes

- Resolução de problemas

- Trabalhos de laboratório

- Trabalhos de grupo

- Trabalhos de pesquisa

- Projetos
Ill,

Avaliação

- Exame no final do módulo

- Testes sumativos

- Avaliação de trabalhos práticos

- Apresentação de um tema

- Apresentação oral

- Relatórios

- Portefólio

Fonte: adaptado de Kennedy (2007).

Tendo em conta os pressupostos referidos, a legislação e a política de formação da instituição de ensino superior onde o curso está a ser implementado com o curso de técnico superior profissional de desenvolvimento de software pretende-se que os futuros técnicos superiores profissionais adquiram competências para responder a problemas e desafios do mundo real, bem como à realização de projetos com a comunidade. O curso é desenvolvido em quatro semestres letivos de igual duração e constituído por 120 ECTS, sendo 3 da área da Matemática e 117 da área das Ciências Informáticas. Salientando, ainda, que nos cursos de engenharia é importante considerar os objetivos, os conteúdos e os resultados de aprendizagem que os estudantes devem alcançar no curso.

A criação do curso técnico superior de desenvolvimento de software obedeceu a três tópicos principais: resultados de aprendizagem, nos quais se enfatiza o que 
se espera que cada formando seja capaz de realizar após a conclusão do curso; módulos curriculares, considerados necessários para dotar os estudantes das competências que permitam o desenvolvimento dos projetos a implementar; projetos implementados no curso, os quais serão desenvolvidos com metodologias de aprendizagem baseadas em projetos.

\section{Resultados de aprendizagem}

O técnico superior profissional em desenvolvimento de software deve ser capaz de efetuar a modelação e o levantamento de requisitos de um projeto de software e de desenvolver aplicações para ambientes desktop, web e móvel que deem resposta a desafios das empresas e das instituições.

Os resultados de aprendizagem foram definidos tendo em conta os níveis e interpretação de competências sugeridos por Kennedy (2007), do menos complexo para o mais complexo: conhecimento - o conhecimento pode ser definido como a capacidade de recuperar ou relembrar factos sem a necessidade de os perceber; compreensão - habilidade de entender e interpretar a informação aprendida; aplicação - capacidade de aplicar conhecimento adquirido em novas situações, nomeadamente, apresentar ideias e conceitos de modo a resolver problemas; análise capacidade de decompor informação em componentes, identificar inter-relações, ideias e compreender a estrutura organizacional; síntese - capacidade de conjugar partes e constituir o todo; avaliação - capacidade de apreciar o valor dos materiais para um determinado fim.

Pelo exposto, os resultados de aprendizagem esperados para os estudantes que concluam o curso de Desenvolvimento de Software, são:

- efetuar, sob orientação, o levantamento de requisitos de um projeto de desenvolvimento de software;

- implementar bases de dados aplicadas a um contexto específico;

- desenvolver aplicações informáticas em ambiente desktop;

- desenvolver aplicações web baseadas em arquiteturas multicamada;

- implementar aplicações para dispositivos móveis recorrendo a uma framework;

- implementar aplicações baseadas em módulos de redes de sensores e dispositivos conectáveis;

- utilizar sistemas de gestão de versões para desenvolvimento colaborativo de software; 
- gerir aplicações para ambiente desktop, web ou dispositivos móveis, incluindo instalação, configuração, testes e manutenção;

- usar técnicas eficazes de comunicação, de relacionamento interpessoal e de motivação no apoio aos utilizadores, através de produção de documentação técnica e recurso a múltiplos meios de comunicação, integrando texto, vídeo e imagem;

- contribuir para a resolução de problemas, através da reflexão e de uma atitude crítica, da criatividade e inovação, da valorização do trabalho em equipa e da comunicação adequada a diferentes públicos, em contexto de aprendizagem ao longo da vida.

Os resultados de aprendizagem foram definidos em termos das competências que os estudantes devem possuir após a conclusão do curso, no entanto não podem ser considerados estanques ou definitivos, pois com a evolução tecnológica e social em cada período de tempo, geralmente muito curto, surgem novos problemas, novos desafios e, consequentemente, a necessidade de novas competências.

\section{Módulos curriculares}

O curso de técnico superior profissional em desenvolvimento de software tem por missão a implementação de um modelo educativo profissionalmente orientado e assente na aprendizagem baseada na prática, tendo por base problemas e desafios do mundo real e a realização de projetos com a comunidade, os quais são desenvolvidos no âmbito das unidades curriculares de Projeto Integrado e de Estágio.

O plano de estudos está organizado em dois anos letivos, distribuídos por quatro semestres, sendo os $1 .^{\circ}$ e $2 .^{\circ}$ semestres administrados no $1 .^{\circ}$ ano e os $3 .^{\circ}$ e $4 .^{\circ}$ semestres administrados no $2 .^{\circ}$ ano.

Seguem-se as unidades curriculares do curso associadas ao respetivo semestre:

- 1. - Semestre: Fundamentos de Desenvolvimento de Software, Introdução à Programação, Introdução aos Sistemas Informáticos, Introdução às Bases de Dados, Matemática, Modelação de Sistemas de Software, Programação, Estágio I, Projeto Integrado I;

- 2. Semestre: Administração de Sistemas, Algoritmos e Estruturas de Dados, Conceção de Interfaces Gráficas, Desenvolvimento Colaborativo de Software, Desenvolvimento de Aplicações, Implementação e Administração de Bases de Dados, Estágio II, Projeto Integrado II; 
- 3.․ Semestre: Introdução às Redes de Dados, Programação para a Web Cliente, Programação para a Web - Servidor, Publicação e Administração Web, Sistemas de Gestão de Conteúdos, Estágio III, Projeto Integrado III;

- 4..$^{\circ}$ Semestre: Computação Móvel, Internet das Coisas, Programação de Dispositivos Móveis, Programação de Serviços Web, Estágio IV, Projeto Integrado IV.

Em termos de número de ECTS cada unidade curricular (administrada somente na instituição de ensino) é de 3 ECTS, cada projeto integrado (administrada na instituição de ensino com ligação à comunidade) é de 6 ECTS, os estágios (administrados na instituição de ensino com ligação à comunidade) são de duração variada em termos de ECTS, sendo Estágio I - 3 ECTS, Estágio II - 6 ECTS, Estágio III - 9 ECTS e Estágio IV - 12 ECTS.

Segue-se uma breve referência aos objetivos, em função das competências esperadas para o estudante, subjacentes às unidades curriculares que constituem o plano de estudos.

Unidades curriculares administradas exclusivamente na instituição de ensino

- Fundamentos de Desenvolvimento de Software: conhecer a importância dos Sistemas de Informação, das metodologias de desenvolvimento de software, da análise de requisitos, da modelação de processos, e da gestão de projeto de desenvolvimento de software;

- Introdução à Programação: conhecer os elementos fundamentais e as construções básicas para o desenvolvimento de programas de computador e compreender conceitos elementares de linguagens de programação orientadas ao objeto;

- Introdução aos Sistemas Informáticos: adquirir conhecimentos essenciais da arquitetura e funcionamento dos sistemas computacionais e dos sistemas operativos, adquirir know-how que permita realizar tarefas básicas de montagem e manutenção de computadores, bem como de instalação, configuração e exploração de sistemas operativos de tipo desktop;

- Introdução às Bases de Dados: usar técnicas e ferramentas de modelação de conceitos de negócio, de desenho de processos de negócio, de modelação e normalização de dados; 
- Matemática: desenvolver competências matemáticas essenciais para a abordagem dos conteúdos dos restantes módulos do CTeSP, nomeadamente, módulos de Bases de Dados e de Programação, e para a resolução dos problemas que ocorrem ao longo do desenvolvimento e evolução dos projetos propostos no CTeSP;

- Modelação de Sistemas de Software: conhecer ferramentas e técnicas fundamentais de modelação UML no contexto do desenvolvimento de software;

- Programação: conhecer conceitos associados a linguagens de programação orientadas ao objeto, desenhar e relacionar classes por herança, associação e agregação, implementar programas de computador com base em linguagens orientadas ao objeto;

- Administração de Sistemas: adquirir know-how que permita realizar tarefas de administração de sistemas autónomos (desktops ligados à rede, com autonomia funcional), explorar ferramentas básicas de virtualização (de tipo hosted);

- Algoritmos e Estruturas de Dados: implementar estruturas de dados e algoritmos de manipulação de estruturas de dados genéricas, medir a complexidade de algoritmos;

- Conceção de Interfaces Gráficas: apresentar as principais questões associadas à ergonomia na interface humano-computador, conhecer os princípios de usabilidade e acessibilidade em interfaces gráficas, adquirir conhecimentos sobre o design gráfico digital, conhecer ferramentas disponíveis para o desenho de interfaces gráficas;

- Desenvolvimento Colaborativo de Software: conhecer o princípio de funcionamento dos sistemas de gestão de versões, utilizar sistemas de gestão de versões em projetos de software, colaborar com outros programadores em projetos de desenvolvimento de software, recorrendo a sistemas de gestão de versões;

- Desenvolvimento de Aplicações: desenvolver aplicações informáticas em ambiente desktop gráfico baseado em eventos;

- Implementação e Administração de Bases de Dados: adquirir conceitos fundamentais de bases de dados, manusear sistemas, técnicas e ferramentas de administração, gestão, e implementar bases de dados; 
- Introdução às Redes de Dados: descrever a arquitetura, a estrutura, as funções, os componentes e os modelos das redes de computadores (incluindo conceitos de meio físico, Ethernet e endereçamento IP), desenvolver competências adequadas que permitam construir LANs simples, executar configurações básicas em routers e switches e implantar esquemas de endereçamento IP;

- Programação para a Web - Cliente: conhecer princípios de desenvolvimento de páginas web em HTML, formatar páginas web, conhecer a programação do lado do cliente e modos de desenvolvimento de sítios web responsivos;

- Programação para a Web - Servidor: desenvolver aplicações informáticas na vertente do servidor utilizando uma framework;

- Publicação e Administração Web: adquirir competências para a instalação e configuração de sistemas Linux com a pilha de desenvolvimento apache2/ nginx PHP e MySQLl, configuração de virtualhosts e tarefas de publicação, atualização, backup e restauro de soluções web, utilizar serviços de alojamento partilhados e dedicados;

- Sistemas de Gestão de Conteúdos: identificar as componentes de um Sistema de Gestão de Conteúdos (CMS), instalar e configurar um CMS, implementar um sítio web, criar um template adequado e a estrutura de navegação, e adicionar conteúdo no CMS, desenvolver plug-ins para extensão das funcionalidades de um CMS;

- Computação Móvel: conhecer as principais arquiteturas dos dispositivos móveis, conhecer as principais frameworks para desenvolvimento de dispositivos móveis, conhecer as formas de interação dos utilizadores com dispositivos móveis, adquirir conhecimentos de programação de aplicações móveis;

- Internet das Coisas: compreender a Internet e sua evolução até a Internet das Coisas (Internet of Things - IoT), construir modelos e protótipos de soluções contendo componentes IoT, apresentar casos práticos contendo IoT da vida particular/doméstica ou empresarial;

- Programação de Dispositivos Móveis: desenvolver competências na implementação de aplicações para ambientes móveis, desenvolver aplicações orientadas a serviços, integrar recursos de dispositivos móveis em aplicações, publicar e gerir aplicações móveis;

- Programação de Serviços Web: implementar arquiteturas baseadas em serviços e integrá-los em diferentes tipos de aplicações. 
Projetos integrados - unidades curriculares administradas na instituição de ensino com ligação à comunidade

Os projetos, tais como as restantes unidades curriculares, foram desenvolvidos no sentido de responderem aos resultados de aprendizagem esperados para o curso. No entanto, pelas suas características na utilização do conhecimento construído nas restantes unidades curriculares, bem como do envolvimento com as empresas, instituições e comunidade, destacam-se os objetivos em função do estudante e a sua tipologia.

Objetivos dos projetos em função dos estudantes:

- Projeto Integrado I: selecionar metodologias de investigação de acordo com a análise a efetuar, estruturar e redigir relatórios técnicos, estruturar e realizar apresentações e comunicações, propor soluções criativas para problemas da comunidade no âmbito do desenvolvimento de soluções de software, integrando conhecimentos de análise de requisitos, modelação de software e modelação de bases de dados e aplicar metodologias e ferramentas CASE e de gestão de projetos;

- Projeto Integrado II: propor soluções para problemas da comunidade no âmbito do desenvolvimento de soluções de software, integrando conhecimentos de programação web (cliente e servidor), publicação web e sistemas de gestão de conteúdos;

- Projeto Integrado III: propor soluções para problemas da comunidade integrando conhecimentos de programação web (cliente e servidor), publicação web e sistemas de gestão de conteúdos;

- Projeto Integrado IV: desenvolver projetos integrados de desenvolvimento de software em articulação com a comunidade, integrando conhecimentos de desenvolvimento para dispositivos móveis, Internet das Coisas e programação de serviços web.

O desenvolvimento dos projetos é sequencial, um em cada semestre, correspondendo o projeto I ao $1 .^{\circ}$ semestre, o projeto II ao $2 .^{\circ}$ semestre, o projeto III ao $3 .^{\circ}$ semestre, e o projeto IV ao $4 . .^{\circ}$ semestre.

Em todo o acompanhamento e orientação dos projetos estará presente a metodologia de aprendizagem baseada em projetos, nomeadamente no modo com se associa a teoria à prática, bem como no respeito e incentivo pela autonomia do estudante e pelo papel que tem na construção do seu próprio conhecimento. 
Estágios - unidades curriculares administradas na instituição de ensino com ligação à comunidade

Como já foi referido, o curso técnico superior profissional de desenvolvimento de software inclui no seu plano de estudos quatro estágios de duração crescente do primeiro para o último, todos eles implicam uma elevada articulação com as empresas ou instituições.

Com a integração dos estágios Estágio I, Estágio II, Estágio III e Estágio IV a instituição de ensino pretende:

- dar a conhecer aos estudantes as empresas e instituições, bem como as suas reais necessidades;

- promover a cooperação bidirecional entre a instituição de ensino e a comunidade;

- inserir os estudantes no contexto real de trabalho;

- integrar o projeto educativo com a realização de projetos e resolução de problemas da comunidade;

- otimizar o tempo e a eficácia da permanência dos estudantes na comunidade;

- promover a criatividade e a capacidade de iniciativa dos estudantes. Com a conclusão dos estágios, pretende-se que o estudante seja capaz:

- aplicar, em atividades praticas, conhecimentos adquiridos e novos conhecimentos apreendidos;

- evidenciar capacidade de análise dos problemas e espirito critico;

- desenvolver trabalho em equipa e tolerância a pressão;

- utilizar competências especificas do curso na sua atividade profissional;

- gerir atividades, no quadro das orientações pré-estabelecidas (sob supervisão geral), assumindo responsabilidade pelas suas realizações e com autonomia de decisão e resolução de problemas de natureza técnica.

A referência a cada unidade curricular de um curso e respetivos objetivos quer em função da instituição de ensino, quer em função do estudante poderão ajudar a compreender as finalidades do curso, e as estratégias utilizadas na sua implementação, permitindo em cada momento avaliar a sua importância e atualização. 


\section{Considerações finais}

É comum afirmar-se que não há melhor prática do que uma boa teoria. Assim, não é possível desenvolver cursos tecnicamente ricos, que respondam às necessidades e desafios das comunidades sem existirem teorias consistentes que os suportem. Por outro lado, as teorias sem a ligação às pessoas reais e às organizações dificilmente poderão responder aos desafios de cada época e de cada comunidade.

Neste artigo partiu-se do pressuposto que as tecnologias de informação e comunicação são essenciais para o desenvolvimento da sociedade, defendendo-se para o processo de ensino e aprendizagem a utilização de metodologias ativas associadas à prática, com destaque para a aprendizagem baseada em projetos. Apresentou-se a caracterização dos cursos de técnico superior profissional e, como exemplo, o modelo de um curso superior profissional de desenvolvimento de software, ministrado numa instituição portuguesa de ensino superior público.

Defendeu-se, ainda, que a aprendizagem baseada em projetos pode ajudar os estudantes a desenvolverem competências altamente tecnológicas e eficazes que contribuam para a resolução de problemas complexos e para responder aos desafios do século XXI.

O curso de técnico superior profissional de desenvolvimento de software que foi objeto de reflexão neste artigo, tem a duração de dois anos letivos e encontra-se no 2. ano de funcionamento, pelo que ainda não foi possível proceder à sua avaliação. No entanto, as opiniões obtidas no contexto de funcionamento do curso, de estudantes, professores, e responsáveis das empresas onde já foram realizados estágios, indiciam apreciações muito positivas sobre o curso e o seu funcionamento, assim como, comparando as médias das classificações das unidades curriculares deste curso, com as médias de unidades curriculares de outros cursos com conteúdos idênticos, as do curso de técnico superior de desenvolvimento de software têm sido mais elevadas.

Como perspetivas futuras espera-se proceder à avaliação do curso, utilizando instrumentos adequados, nomeadamente em termos do grau de satisfação dos estudantes com o curso e com as metodologias utilizadas, competências adquiridas, empregabilidade, e grau de satisfação das entidades empregadoras destes profissionais. 
1 Iniciativa Portugal INCoDe.2030. Disponível em: <http://www.incode2030.gov.pt/>. Acesso em: 05 out. 2018.

\section{Referências}

BENDER, W. Project-based learning: differentiating instruction for the 21st century. Corwin: Thousand Oaks, 2012.

BINKLEY, M. et al. Defining twenty-first century skills. In: GRIFFIN, P.; MCGAW, B.; CARE, E. (Org.). Assessment and teaching of 21st century skills: methods and approach. Dordrecht: Springer, 2012. p. 17-66.

BOYACI, S.; ATALAY, N. A scale development for 21st Century skills of primary school students: a validity and reliability study. International Journal of Instruction, Ankara, v. 9, n. 1, p. 133-148, 2016.

CAVANAUGH, C. Project-based learning in undergraduate educational technology. In: SOCIETY FOR INFORMATION TECHNOLOGY \& TEACHER EDUCATION INTERNATIONAL CONFERENCE. Proceedings... Atlanta, 2004. p. 2010-2016.

CHOUNTA, I.; MANSKE, S.; HOPPE, H. From Making to Learning: introducing Dev Camps as an educational paradigm for Re-inventing Problem-based Learning. International Journal of Educational Technology in Higher Education, v. 14, n. 1, p. 14-21, 2017.

COMISSÃO EUROPEIA. Uma nova agenda de competências para a Europa. 2016. Disponível em: <https://eur-lex.europa.eu/legal-content/pt/txt/pdf/?uri=celex:52016dc0381 \&from=en>. Acesso em: 05 out. 2018.

COMISSÃO EUROPEIA. Europe's Digital Progress Report 2017. 2017. Disponível em: <https:// ec.europa.eu/digital-single-market/en/news/europes-digital-progress-report-2017>. Acesso em: 05 out. 2018.

DRAGOUMANOS, S.; KAKAROUNTAS, A.; FOUROU, T. Young technology entrepreneurship enhancement based on an alternative approach of project-based learning. In: GLOBAL ENGINEERING EDUCATION CONFERENCE (EDUCON), Proceedings... 2017. p. 351-358.

FLORIDA STATE UNIVERSITY. Instruction at FSU: a guide to teaching and learning practices. Office of Distance learning, 7th edition. 2011. Disponível em: <https://distance.fsu.edu/instructors/instruction-fsu-guide-teaching-learning-practices>. Acesso em: 05 out. 2018.

ITURREGI, A. et al. Work in Progress: Project-based learning for electrical engineering. In: IEEE GLOBAL ENGINEERING EDUCATION CONFERENCE (EDUCON). Proceedings... Athens, 2017. p. 464-467.

KENNEDY, D. Writing and Using Learning Outcomes - a Practical Guide. Cork: University College Cork, 2007.

KRAJCIK, J.; BLUMENFELD, P. Project-Based Learning. In: SAWYER, R. (Ed). The Cambridge Handbook of the Learning Sciences. Cambridge: Cambridge University Press, 2006. 
LAAR, E. et al. The relation between 21st-century skills and digital skills: a systematic literature review. Computers in Human Behavior, n. 72, p. 577-588, 2017.

LARMER, J.; MERGENDOLLER, J.; BOSS, S. Setting the standard for project based learning. Alexandria, VA: ASCD, 2015.

MAHASNEH, A.; ALWAN, A. The Effect of Project-Based Learning on Student Teacher Self-efficacy and Achievement. International Journal of Instruction, v. 11, n. 3, p. 511-524, 2018.

MARTINS, V. et al. Problem-based Learning Methodology Applied within a Data Network Infrastructure Design Course: a Real Case Implementation. In: IBERIAN CONFERENCE ON INFORMATION SYSTEMS AND TECHNOLOGIES, CISTI 2017, 12. Proceedings... 2017. p. 1891-1896.

MARX, R. et al. Inquiry $\square$ based science in the middle grades: Assessment of learning in urban systemic reform. Journal of Research in Science Teaching, Michigan, n. 41, p. 1063-1080, 2004.

NIEH, T.; CHOU, J. A Project-based Learning Design for teaching and learning of Mechatronics Engineering, The Riderless Bicycle as an Application. In: IEEE GLOBAL ENGINEERING EDUCATION CONFERENCE (EDUCON). Proceedings... 2018. p. 251-256.

REQUIES, J. et al. Evolution of Project-Based Learning in Small Groups in Environmental Engineering Courses. Journal of Technology and Science Education, Terrassa, v. 8, n. 1, p. 45-62, 2018.

RIBEIRO, L.; MIZUKAMI, M. Uma Implementação da Aprendizagem Baseada em Problemas (PBL) na Pós-Graduação em Engenharia sob a Ótica dos Alunos. Semina: Ciências Sociais e Humanas, Londrina, v. 25, p. 89-102, 2004.

RIVET, A.; KRAJCIK, J. Achieving Standards in Urban Systemic Reform: An Example of a Sixth Grade Project-Based Science Curriculum. Journal of Research in Science Teaching, v. 41, n. 7, p. 669-692, 2004.

SEMANA, L.; HAUSMANNB, R.; BEZERRA, E. On the students' perceptions of the knowledge formation when submitted to a Project-Based Learning environment using web applications. Computers \& Education, v. 117, p. 16-30, 2018.

VOOGT, J.; ROBLIN, N. A comparative analysis of international frameworks for 21st century competences: Implications for national curriculum policies. Journal of Curriculum Studies, London, v. 44, n. 3, p. 299-321, 2012.

WORLD ECONOMIC FORUM. The Future of Jobs. Global Challenge Insight Report. 2016. Disponível em: <http://reports.weforum.org/future-of-jobs-2016>. Acesso em: 05 out. 2018. 